\title{
Antimicrobial lubricant formulations containing poly(hydroxybenzene)-trimethoprim conjugates synthesized by tyrosinase
}

\author{
Idalina Gonçalves • Cláudia M. Botelho • Ana Teixeira • \\ Ana S. Abreu • Loïc Hilliou • Carla Silva • Artur Cavaco-Paulo
}

Received: 22 January 2015 /Revised: 23 February 2015 / Accepted: 24 February 2015 / Published online: 12 March 2015

(C) Springer-Verlag Berlin Heidelberg 2015

\begin{abstract}
Poly(hydroxybenzene)-trimethoprim conjugates were prepared using methylparaben as substrate of the oxidative enzyme tyrosinase. MALDI-TOF MS analysis showed that the enzymatic oxidation of methylparaben alone leads to the poly(hydroxybenzene) formation. In the presence of trimethoprim, the methylparaben tyrosinase oxidation leads poly(hydroxybenzene)-trimethoprim conjugates. All of these compounds were incorporated into lubricant hydroxyethyl cellulose/glycerol mixtures. Poly(hydroxybenzene)-trimethoprim conjugates were the most effective phenolic structures against the bacterial growth reducing by 96 and $97 \%$ of
\end{abstract}

Idalina Gonçalves and Cláudia M. Botelho contributed equally to this work.

Electronic supplementary material The online version of this article (doi:10.1007/s00253-015-6509-1) contains supplementary material, which is available to authorized users.

I. Gonçalves • C. M. Botelho • A. Teixeira • A. S. Abreu • C. Silva •

A. Cavaco-Paulo $(\triangle)$

CEB - Centre of Biological Engineering, University of Minho,

4710-057 Braga, Portugal

e-mail: artur@deb.uminho.pt

\section{Hilliou}

Department of Polymer Engineering, University of Minho, 4800-058 Guimarães, Portugal

Present Address:

A. S. Abreu

IPC - Institute of Polymer and Composites/I3N- Institute of Nanostructures, Nanomodelling and Nanofabrication, University of Minho, 4800-058 Braga, Portugal

Present Address:

C. Silva

3B's Research Group, Biomaterials, Biodegradables and

Biomimetics, University of Minho, Headquarters of the European

Institute of Excellence on Tissue Engineering and Regenerative

Medicine, Ave Park, S. Cláudio de Barco, Taipas, 4806-

909 Guimarães, Portugal
Escherichia coli and Staphylococcus epidermidis suspensions, respectively (after $24 \mathrm{~h}$ ). A novel enzymatic strategy to produce antimicrobial poly(hydroxybenzene)-antibiotic conjugates is proposed here for a wide range of applications on the biomedical field.

Keywords Methylparaben · Trimethoprim · Tyrosinase · Poly(hydroxybenzene) $\cdot$ Conjugates $\cdot$ Antimicrobial

\section{Introduction}

Catheter-related infections (CRIs) have been one of the most problematic issues in the management of nosocomial infections (Kumon et al. 2001). The infection usually occurs in indwelling devices following three possible routes, namely bacterial colonization onto the outer/inner catheter surface, bloodstream or urethral fluids infection, and skin exit site infection (Alkubati et al. 2015). Despite the high rates of CRIs, these have been considered to be preventable nosocomial infections since various practices as easy-to-perform rules for hygiene viz. usage of sterile barriers during catheter insertion, masks, gowns and caps have proven to be effective for avoiding early CRIs (Capdevila 1998). Hereupon, multiple strategies for the CRIs prevention have been emphasized which include educating and training health care workers who insert and maintain the indwelling catheters. The application of locking formulations containing bioactive compounds as antibiotics (heparin, vancomycin) as well as the use of antimicrobial lubricants attempts to block the catheter colonization and subsequent development of CRIs (Frasca et al. 2010; Snaterse et al. 2010). An antimicrobial formulation can be defined as a system containing an active molecule along with some inert ingredients as gelling/thickening (e.g., hydroxyethyl cellulose) and lubricating (e.g., glycerol) agents 
(Egbaria and Friedman 1990; Garg et al. 2001). Preservative agents such as parabens are also often present in pharmaceutical formulations in order to inhibit the bacterial growth during storage (Naik and Nandibewoor 2013; Wang and Chang 1998). Their biological action is generally related to their inhibitory effects on membrane transport and mitochondrial function processes (Naik and Nandibewoor 2013; Soni et al. 2005). Parabens have an ester group derived from $p$ hydroxybenzoic acid at the C-4 position. These are stable compounds in a wide $\mathrm{pH}$ range and do not have a perceptible odor or taste. Moreover, they do not cause changes on the product consistency, and most of all, they are safe, i.e., parabens evidence low frequency of sensitization, low toxicity levels, and do not evidence carcinogenic activity (Błędzka et al. 2014; Mielech-Łukasiewicz et al. 2011; Soni et al. 2002). Amongst the most common parabens used, methylparaben, propylparaben, and butylparaben possess a broad spectrum of antibacterial activity that is directly related to the alkyl chain length; however, the last two compounds are less soluble than methylparaben. In addition, gram-positive strains revealed to be more sensitive to methylparaben than gram-negative bacteria (Soni et al. 2002). Nevertheless, different parabens combinations have been reported to promote a synergistic effect on gram-negative bacteria (Charnock and Finsrud 2007).

In this work was studied the enzymatic oxidation of methylparaben using tyrosinase as biocatalyst aiming to exploit its antimicrobial properties, maintaining its known preservative behavior and therefore expanding its application range. The monohydric phenol on the methylparaben structure makes it a potential substrate for tyrosinase (EC 1.14.18.1, monophenols, o-diphenol: oxygen oxidoreductase). Tyrosinase is capable to oxidize both monophenols to $o$-diphenols and $o$-diphenols to the corresponding $o$-quinones. The quinones formed are highly reactive compounds that can undergo nucleophilic attack, i.e., nonenzymatic reactions, by other compounds as phenolic groups, amino acids, peptides, or proteins (Sousa et al. 2009). The rapid polymerization of $o$-quinones leads to polyphenols with increased antimicrobial properties than the corresponding monomer. Moreover, these highly hydroxylated compounds have great biological activities such as anti-inflammatory, anti-allergic, anti-thrombosis, antioxidant, and antimicrobial properties (Alberto et al. 2011). As described previously by Gonçalves et al. (Gonçalves et al. 2015), the conjugation of polyphenols with antibiotics had a synergistic effect against bacterial growth. The oxidation process involved a two-stage method in which the monomer (catechin) was chemically conjugated with antibiotics and further polymerized through laccase action.

The main goal of this work is to produce novel antimicrobial polyphenolic-antibiotic conjugates by a one-step method and compare it's efficiency with a previously produced poly(catechin)-trimethoprim conjugate. Herein, the poly(hydroxybenzene)-trimethoprim conjugates were produced through in situ enzymatic oxidation of methylparaben using tyrosinase as biocatalyst. The poly(catechin)-trimethoprim conjugates were obtained following the Gonçalves et al. (2015) methodology. Both polyphenolic-antibiotic conjugates were incorporated into a newly optimized lubricant matrix envisaging a topical application and the antimicrobial activity of each formulation was evaluated against gram-negative and gram-positive bacteria.

\section{Material and methods}

\section{Material}

Tyrosinase (EC 1.14.18.1) from mushroom (3130 U/mg $\mathrm{molid}_{\text {sid }}$, methylparaben, catechin hydrate, trimethoprim, chloroform, methanol, sodium acetate trihydrate, ethanol, trifluoroacetic acid, acetonitrile, water, bovine serum albumin (BSA), 2,2'azinobis-(3-ethylbenzothiazoline-6-sulfonic acid) (ABTS), $N$, $\mathrm{N}$-disuccinimidyl carbonate (DSC), 4-(dimethylamino)pyridine (DMAP), hydroxyethyl cellulose (HEC), glycerol, and Mueller-Hinton (MH) broth and agar were purchased from Sigma-Aldrich (Spain). Laccase (EC 1.10.3.2) from ascomycete Myceliophthora thermophila $(17 \mathrm{~g}$ protein/L, $410 \mathrm{U} / \mathrm{mL}$ at $50{ }^{\circ} \mathrm{C}$ ), Novozym ${ }^{\circledR} 51003$, was obtained from Novozymes (Denmark).

\section{Bacterial strains}

The bacterial strains, Escherichia coli HB101 and Staphylococcus epidermidis ATCC 35983, were used from the collection of University of Minho (Braga, Portugal).

\section{Methods}

\section{Enzymatic synthesis of poly(hydroxybenzene) using} methylparaben as tyrosinase substrate

In a first stage, methylparaben was oxidized by tyrosinase using different concentrations of substrate $(1,5$, and $10 \mathrm{mM})$ and a fixed enzyme amount $(20 \mathrm{U} / \mathrm{mL})$. Methylparaben was dissolved in $0.1 \mathrm{M}$ sodium phosphate buffer $(\mathrm{pH} 7)$ and incubated with tyrosinase at $30^{\circ} \mathrm{C}$ for $24 \mathrm{~h}$ under orbital agitation (90 rpm). Posteriorly, the optimum tyrosinase dosage was investigated by varying the enzyme concentration $(10,20,30$, 50 , and $100 \mathrm{U} / \mathrm{mL}$ ) and fixing the methylparaben concentration previously determined. The resulting polyphenol, obtained using the best experimental conditions, was freeze-dried under vacuum conditions for $24 \mathrm{~h}$ and stored at dry and dark conditions. 
In situ enzymatic synthesis of poly(hydroxybenzene) -trimethoprim conjugates through tyrosinase action

For the in situ enzymatic synthesis of poly(hydroxybenzene)trimethoprim conjugates, 1 eq. of trimethoprim previously dissolved in methanol was added to the methylparaben solution prepared in $0.1 \mathrm{M}$ sodium phosphate buffer ( $\mathrm{pH} 7)$. The reaction was performed using $50 \mathrm{U} / \mathrm{mL}$ of tyrosinase at $30^{\circ} \mathrm{C}$ for $24 \mathrm{~h}$ with $90 \mathrm{rpm}$. The obtained polyphenol was freezedried under vacuum conditions over $24 \mathrm{~h}$.

\section{Enzymatic synthesis of poly(catechin) using catechin as laccase substrate}

According to literature (Gonçalves et al. 2014), a solution of $10 \mathrm{mM}$ of catechin monomer was oxidized by laccase (2 U/ $\mathrm{mL}$ ) to produce poly(catechin). Catechin was previously dissolved in methanol and further diluted with $0.1 \mathrm{M}$ sodium acetate buffer ( $\mathrm{pH} 5)$. The reaction was carried out at $50{ }^{\circ} \mathrm{C}$ and $70 \mathrm{rpm}$ for $24 \mathrm{~h}$. The polymer was obtained by centrifugation at $4000 \mathrm{rpm}$ for $10 \mathrm{~min}$ at $4{ }^{\circ} \mathrm{C}$ using an Allegra ${ }^{\circledR} \mathrm{X}-15 \mathrm{r}$ Centrifuge (Beckman Coulter International) and washed three times with distilled water.

\section{In situ enzymatic synthesis of poly(catechin)-trimethoprim conjugates through laccase action}

According to Gonçalves et al. (2015), the enzymatic synthesis of poly(catechin)-trimethoprim conjugates followed a twostep pathway: (1) chemical conjugation of catechin with trimethoprim and (2) enzymatic polymerization of catechintrimethoprim conjugates through laccase oxidation. In the first stage, the conjugation was initiated by the activation of hydroxyl groups present at the catechin structure by mixing $0.03 \mathrm{M}$ of phenol with DSC $(0.1 \mathrm{M})$ and DMAP $(0.1 \mathrm{M})$ in 1,4-dioxane anhydrous under a nitrogen atmosphere for $24 \mathrm{~h}$. Subsequently, for catechin-trimethoprim conjugation, 1 eq. of TMP was added to the activated catechin previously dissolved in methanol, and the process remained overnight at room temperature with stirring. Furthermore, the enzymatic oxidation of catechin or catechin-trimethoprim conjugates was carried out using $2 \mathrm{U} / \mathrm{mL}$ of laccase at $50{ }^{\circ} \mathrm{C}$ for $2 \mathrm{~h}$ with $70 \mathrm{rpm}$ under aired conditions. The reaction product was centrifuged at $4{ }^{\circ} \mathrm{C}$ for $15 \mathrm{~min}$, washed with distilled water, and dried at room temperature.

\section{MALDI-TOF MS spectrometry}

The poly(hydroxybenzene) compounds obtained by tyrosinase-catalyzed oxidation were identified through matrix-assisted laser desorption/ionization time-of-flight mass spectrometry (MALDI-TOF MS). The $\alpha$-cyano-4hydroxycinnamic acid (CHCA) was the matrix selected. Five milligrams per milliliter of CHCA was dissolved on a solution of $33 \%(v / v)$ of ethanol, $31 \%(v / v)$ of distilled water, $33 \%(v /$ $v)$ of acetonitrile, and $3 \%(v / v)$ of trifluoroacetic acid. For the sample-matrix preparation, $1.5 \mathrm{mg}$ of sample was dissolved in $20 \mu \mathrm{L}$ of CHCA matrix. A $0.5 \mu \mathrm{L}$ of the sample-matrix solution was placed on FlexiMass ${ }^{\mathrm{TM}}$-DS plates (Kratos analytical, Shimadzu, UK) equipped with a nitrogen laser radiating at $337 \mathrm{~nm}$ and dried at room temperature for $10 \mathrm{~min}$. The analyses were performed on an Axima LNR system (Kratos analytical, Shimadzu, UK). All the spectra were recorded using a linear mode with a delay time of $104 \mathrm{~ns}$, an acceleration voltage of $+20 \mathrm{kV}$, and $0.002 \%$ of guide wire in a mass range from 50 to $5000 \mathrm{Da}$. The resulting peaks were identified using the Shimadzu Biotech software. All the analyses were performed in triplicate.

\section{UV-Visible measurements}

The spectrophotometric changes through polymerization were assessed by UV-visible spectrophotometry at a wavelength between 230 and $700 \mathrm{~nm}$. The equipment used was a Multimode Microplate Reader Synergy ${ }^{\mathrm{TM}} \mathrm{Mx}$ and Gen5 $5^{\mathrm{TM}}$ purchased from Biotek ${ }^{\circledR}$ Instruments, Inc. (USA). Each polyphenolic solution was measured in triplicate.

\section{Lubricant formulation production}

The lubricant formulation production was achieved using a HEC/glycerol matrix prepared in distilled water. Three different HEC/glycerol ratios were tested, namely $2.8: 11.1,2.5: 10$, and 2.1:8.3\%(w/v). The mixture was stirred at room temperature for $24 \mathrm{~h}$.

Incorporation of polyphenolic compounds onto the lubricant formulation

Aiming to introduce the polyphenolic compounds in the lubricant matrix and due to their own low solubility, $2.5 \mathrm{mg}$ of each polymer was dissolved in a volume ratio of 1:4 of solvent phase (ethanol)/aqueous phase (distilled water) and mechanically mixed for approximately $10 \mathrm{~min}$ at room temperature. Then, the obtained solution was sonicated for $30 \mathrm{~min}$ (ultrasound pulses of $8 \mathrm{~s}$ ON and $8 \mathrm{~s}$ OFF at $20 \mathrm{kHz}$ with $40 \%$ of amplitude) to ensure total dissolution. Afterward, the polyphenolic solution was incorporated in the lubricant formulation $(30 \% v / v)$ using a dropwise technique and left under magnetic stirring until it was completely mixed.

Rheological tests

The rheological behavior of each lubricant formulation was evaluated using a rheometer Physica MCR-300 (Anton Paar, Austria) and employing a couette geometry in which the 
material viscosity changes versus the shear rate were evaluated. Ten milliliters of each formulation was placed inside the cylinder of the rheometer, and the flow measurements were carried out at 25 and $37^{\circ} \mathrm{C}$. The shear rate was ranged from 0.01 to $100 / \mathrm{s}$. The results obtained were fitted using the Carreau Yasuda model (Eq. 1):

$\eta=\eta_{\infty}+\left(\eta_{0}-\eta_{\infty}\right)\left[1+(\gamma \lambda)^{a}\right]^{\frac{n-1}{a}}$

where $\eta$ is the viscosity, $\eta_{\infty}$ is the infinite-shear-rate (parameter fixed at 0$), \eta_{0}$ is the Newtonian viscosity, $\dot{\gamma}$ is the shear rate $(1 / s), \lambda$ is the relaxation time (s), $n$ is the power law index, and $a$ is the polydispersity in relaxation times.

\section{Antimicrobial assays}

The antimicrobial activity of each developed polyphenol was assessed against gram-negative (E. coli) and gram-positive (S. epidermidis) bacterial strains following two experimental approaches, namely, optical density (O.D.) measurement and CFUs count in plate. As a preliminary stage, the bacteria were inoculated overnight in Mueller-Hilton broth (MHB) at $37^{\circ} \mathrm{C}$ and $120 \mathrm{rpm}$. Thereafter, the bacteria cells were centrifuged at $9000 \mathrm{~g}$ for $10 \mathrm{~min}$ and washed twice with distilled water. A calibration curve was performed in order to determine the bacterial concentration for different O.D. measured at $640 \mathrm{~nm}$. The cellular suspension was adjusted to a final concentration of $2 \times 10^{5} \mathrm{CFUs} / \mathrm{mL}$ measured by O.D. at the wavelength of $640 \mathrm{~nm}$. Five milliliters of bacterial suspension was mixed with $5 \mathrm{~mL}$ of lubricant and incubated at $37^{\circ} \mathrm{C}$ for $72 \mathrm{~h}$ with $120 \mathrm{rpm}$. The O.D. at $640 \mathrm{~nm}$ was measured every $24 \mathrm{~h}$ in triplicate. For the CFUs count in plate, $100 \mu \mathrm{L}$ of sample was serially diluted with distilled water and three drops of $10 \mu \mathrm{L}$ each were platted onto $\mathrm{MH}$-agar plates. Aiming to explore the antimicrobial durability of each produced polyphenolic compound, the plates were incubated at $37^{\circ} \mathrm{C}$ for 24, 48, and $72 \mathrm{~h}$ and the resulting CFUs counted. Lubricant solutions without any phenolic compound were used as reference samples. The percentage of bacterial reduction considered the CFUs obtained after the inoculation of each tested phenolic lubricant matrix against control samples.

\section{Results}

Enzymatic synthesis of poly(hydroxybenzene) using methylparaben as tyrosinase substrate

The antimicrobial profile of methylparaben was improved through enzymatic oxidation using tyrosinase as biocatalyst. Considering the well-described oxidation of phenolic compounds by this enzyme (Ba et al. 2014; Faccio et al. 2012; Xu and Yang 2013) and the known chemical oxidation of methylparaben (Steter et al. 2014), it proposed four main sequential steps for methylparaben oxidation: (1) nucleophilic addition of the hydroxyl radical to the carbon of the carbonyl group present in the methylparaben structure, followed by the elimination of the methoxyl group $\left(-\mathrm{OCH}_{3}\right)$ leading to the $p$ hydroxybenzoic acid formation (Steter et al. 2014), (2) decarboxylation of the $p$-hydroxybenzoic acid (-COOH) to form phenol units, (3) hydroxylation and oxidation of monophenols to $o$-quinones by tyrosinase action (Decker and Tuczek 2000; Demarche et al. 2012), and (4) nonenzymatic polymerization of $o$-quinones resulting polyphenolic compounds. Another reaction by-product that can be obtained is the benzoic acid derived from the dehydroxylation (-OH) of $p$-hydroxybenzoic acid (Fig. 1).

The formation and nature of polyphenols from tyrosinasecatalyzed reactions using methylparaben as substrate were explored by MALDI-TOF MS. As it was abovementioned, in this work, it was expected that the enzymatic oxidation of methylparaben could give rise to a panoply of structures, namely polymers with different lengths. Table 1 shows the most significant $\mathrm{m} / \mathrm{z}$ peaks representing polymers of hydroxybenzene with a length range from 5 to 37 phenolic units. Considering that each hydroxybenzene unit corresponds to $93 \mathrm{Da}$, it was easily confirmed that compounds containing at least five hydroxybenzene units $(\mathrm{m} / \mathrm{z} 465)$ resulted from the methylparaben oxidation via tyrosinase. Besides, other poly(hydroxybenzene) chains containing various functional groups as methyl esters $(\mathrm{m} / \mathrm{z} 1178)$ and carboxylic acids $(\mathrm{m} / z 2766$ and $\mathrm{m} / \mathrm{z}$ 3696) were also identified by MALDITOF MS analysis which indicate the great variability amongst the formed reaction products.

Aiming to maximize the reaction yield, three methylparaben concentrations were tested, and the UV/ Visible changes at $430 \mathrm{~nm}$ were more pronounced when $5 \mathrm{mM}$ of the substrate was used (Fig. 2a). This concentration was therefore considered as the optimal for the subsequent experiments. Different concentrations of tyrosinase were assessed, and the results indicated the highest absorbance at $430 \mathrm{~nm}$ when $50 \mathrm{mM}$ of enzyme was used (Fig. 2b). For higher enzyme concentrations, the absorbance at $430 \mathrm{~nm}$ reached a plateau meaning that no modifications are observed above this concentration.

In situ synthesis of poly(hydroxybenzene)-trimethoprim conjugates by tyrosinase

The well-described oxidation, biodegradation, and metabolism of trimethoprim (Michael et al. 2012) allowed to conclude that, in the presence of trimethoprim, the methylparaben oxidation by tyrosinase yields poly(hydroxybenzene)-trimethoprim conjugates. During this process, demethylation and/or 


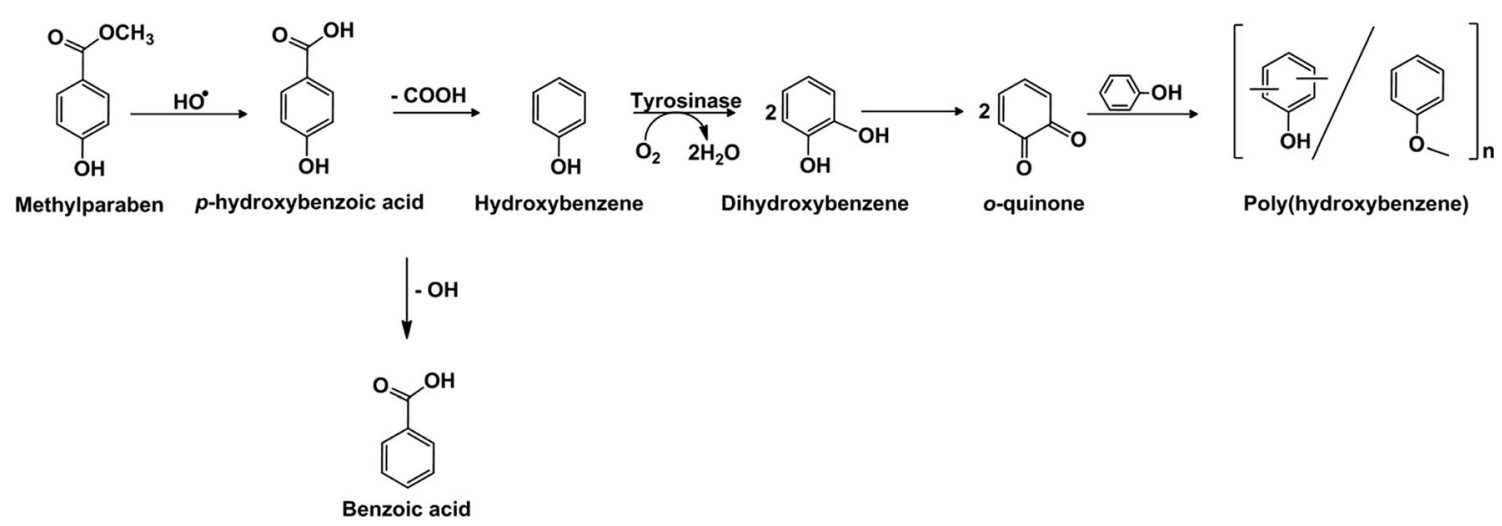

Fig. 1 Schematic representation of enzymatic methylparaben oxidation using tyrosinase as biocatalytic agent

hydroxylation of trimethoprim can promote compounds which maintain the two-ring trimethoprim structure such as the 5-((2,4-diaminopyridimidin-5-yl)methyl)-2,3dimethoxyphenol. Subsequently, the cleavage of these compounds can result in $N$-heteroaromatic derivatives such as the 2,4-diaminopyrimidine-5-carboxylic acid (Fig. 3). Therefore, it is our assumption that both of these products are suitable to react with the poly(hydroxybenzene) chains that resulted from the enzymatic oxidation of methylparaben by tyrosinase giving rise to newly poly(hydroxybenzene) conjugates.

As can be seen in Table 2, the MALDI-TOF MS analysis of the reaction products resulted from the tyrosinase oxidation of methylparaben in the presence of trimethoprim that allowed identifying unchanged antibiotic molecules $(\mathrm{m} / z$ 292) that were not affected by the enzymatic process as well as observing the poly(hydroxybenzene) conjugates formation in which a trihydroxybenzene chain was conjugated with a trimethoprim molecule $(\mathrm{m} / z$ 570) and another hexahydroxybenzene compound was linked to 2,4-diaminopyrimidine-5-carboxilic acid $(\mathrm{m} / \mathrm{z} 715)$, a degradation by-product of trimethoprim.

\section{Development of lubricant formulations}

Three different lubricant formulations were prepared by changing the HEC/glycerol ratio. The rheological properties of each mixture showed a non-Newtonian behavior (Fig. S1). At both temperatures tested, 25 and $37{ }^{\circ} \mathrm{C}$, a nonlinear relationship between the shear rate and the viscosity was observed, and the highest viscosity profile was observed for the most concentrated formulation (2.8:11.1\% HEC/glycerol, $w / v)$ for all the shear rate values tested.

Incorporation of polyphenolic compounds onto the lubricant formulation

As previously mentioned, the two-step enzymatic synthesis of poly(catechin)-trimethoprim via laccase oxidation reported by Gonçalves et al. (2015) was also assessed in this work aiming to compare the antimicrobial efficiency of two polyphenol- antibiotic conjugates. When polyphenolic-trimethoprim conjugates were added into the lubricant formulation, the main difference macroscopically observed was the color change. The initial formulation was colorless. Once the polyphenolic-trimethoprim conjugates were added, the formulation acquired a yellowish color (Fig. S2).

The rheological evaluation of the lubricant-containing conjugates was performed. Figure $\mathrm{S} 3$ shows that, at $37^{\circ} \mathrm{C}$, after the conjugates addition, the non-Newtonian behavior of the lubricant was maintained, being observed a significant decrease of the initial viscosity.

Antimicrobial activity of the lubricant-containing polyphenolic compounds

The antimicrobial efficiency of lubricant formulations containing each developed polyphenolic compound, namely, poly(hydroxybenzene), poly(catechin), poly(hydroxybenzene)trimethoprim, and poly(catechin)-trimethoprim conjugates was evaluated against the $S$. epidermidis and $E$. coli growth. Figure 4 shows that the lubricant containing both poly(hydroxybenzene) or poly(catechin) presented higher antimicrobial activity against E. coli than against $S$. epidermidis, for all the incubation periods tested. Indeed, the reduction of S. epidermidis is relatively low, being more pronounced on the first $48 \mathrm{~h}$ of inoculation. The lubricant-containing polyphenolic-trimethoprim conjugates presented the highest ability to reduce the bacterial growth. This reduction is more evident for poly(hydroxybenzene)-trimethoprim conjugates, especially in the first $24 \mathrm{~h}$ of inoculation, and their action against $S$. epidermidis was slightly more pronounced in comparison with E. coli.

\section{Discussion}

In this work was studied the methylparaben oxidation through tyrosinase, a well-known enzyme able to oxidize monohydric phenols into the corresponding $o$-quinones. The tyrosinase-dependent activation of hydroxybenzenes 
Table 1 MALDI-TOF MS analysis of different polymers formed during enzymatic oxidation of methylparaben through tyrosinase action

Number of monomers Structure

$m / z$

dihydroxybenzene-1,4-dihydroxyphenol

10<smiles>Cc1ccc(Oc2ccc(O)cc2)cc1</smiles>

polyhydroxybenzene-1,4-dihydroxyphenol

11<smiles>COC(=O)c1ccc(Oc2ccc(Oc3ccc(O)cc3)cc2)cc1</smiles>

polyhydroxybenzene-1-hydroxyphenol-4-methylbenzoate

27<smiles>Cc1ccc(Oc2ccc(C(=O)O)cc2)cc1</smiles>

\section{polyhydroxybenzene-1-hydroxyphenol-4-methylbenzoate}

polyhydroxybenzene-1,4-dihydroxybenzoic acid

Fig. 2 Optimization of the experimental conditions for enzymatic oxidation of methylparaben by tyrosinase considering the substrate (a) and tyrosinase (b) concentrations. The procedures were carried out in $0.1 \mathrm{M}$ sodium phosphate buffer (pH 7) at $37^{\circ} \mathrm{C}$ for $24 \mathrm{~h}$
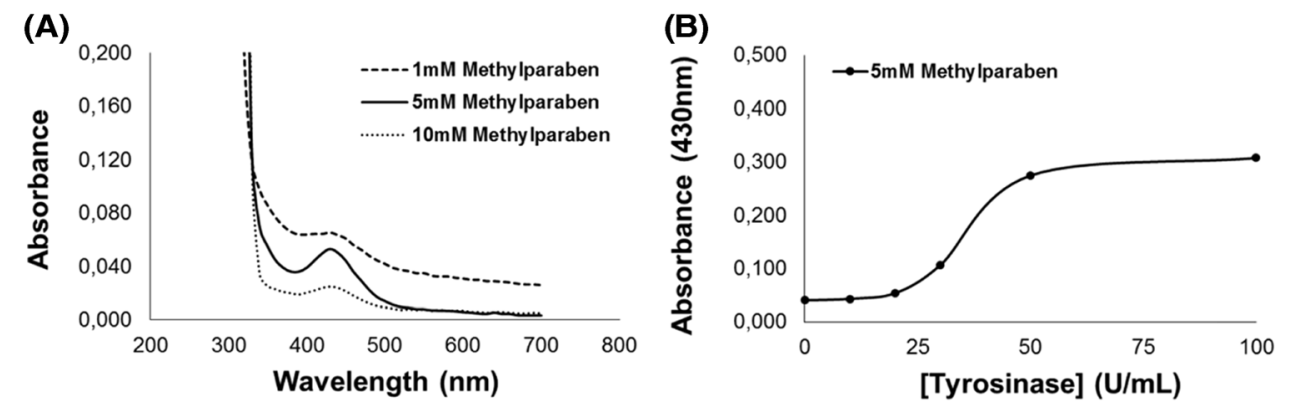
Fig. 3 Reaction products resulting from the trimethoprim cleavage and demethylation/ hydroxylation reactions (Michael et al. 2012)<smiles>COc1cc(Cc2cnc(N)nc2N)cc(OC)c1OC</smiles>

2,4-diaminopyrimidine-5-carboxylic acid

5-((2,4-diaminopyridimidin-5-yl)methyl)-2,3-dimethoxyphenol

yielding reactive compounds including free radicals has also been reported as an important characteristic of this enzyme (Tada et al. 2010, 2014). On the other hand, the in situ generation of radicals has been considered as an effective technology in the degradation of various organic compounds as methylparaben (Steter et al. 2014). Indeed, hydroxyl radicals $(\mathrm{OH})$ are able to react with the carbon of the carbonyl group present in the methylparaben structure and subsequently to eliminate the methoxyl group ($\left.\mathrm{OCH}_{3}\right)$. The resulting $p$-hydroxybenzoic acid can be easily decarboxylated $(-\mathrm{COOH})$ by the $\mathrm{OH}$ insertion yielding monohydric phenol units that are suitable to be oxidized

Table 2 MALDI-TOF MS analysis of different oligomers formed during enzymatic oxidation of methylparaben through tyrosinase action in the presence of trimethoprim

Number of monomers

Trimethoprim

$m / z$<smiles>COc1cc(Cc2cnc(N)nc2N)cc(OC)c1OC</smiles>

Trimethoprim<smiles>COc1cc(Cc2cnc(Nc3ccc(Oc4ccc(O)cc4)cc3)nc2N)cc(OC)c1OC</smiles>

dihydroxybenzene-1-trimethoprim-4-hydroxyphenol
6

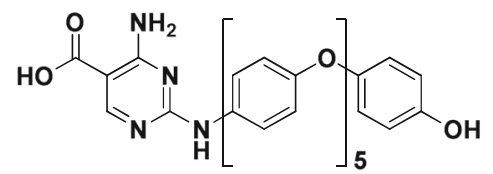

pentahydroxybenzene-1-[2,4-diaminopirimidine-5carboxylic acid]-4-hydroxyphenol 

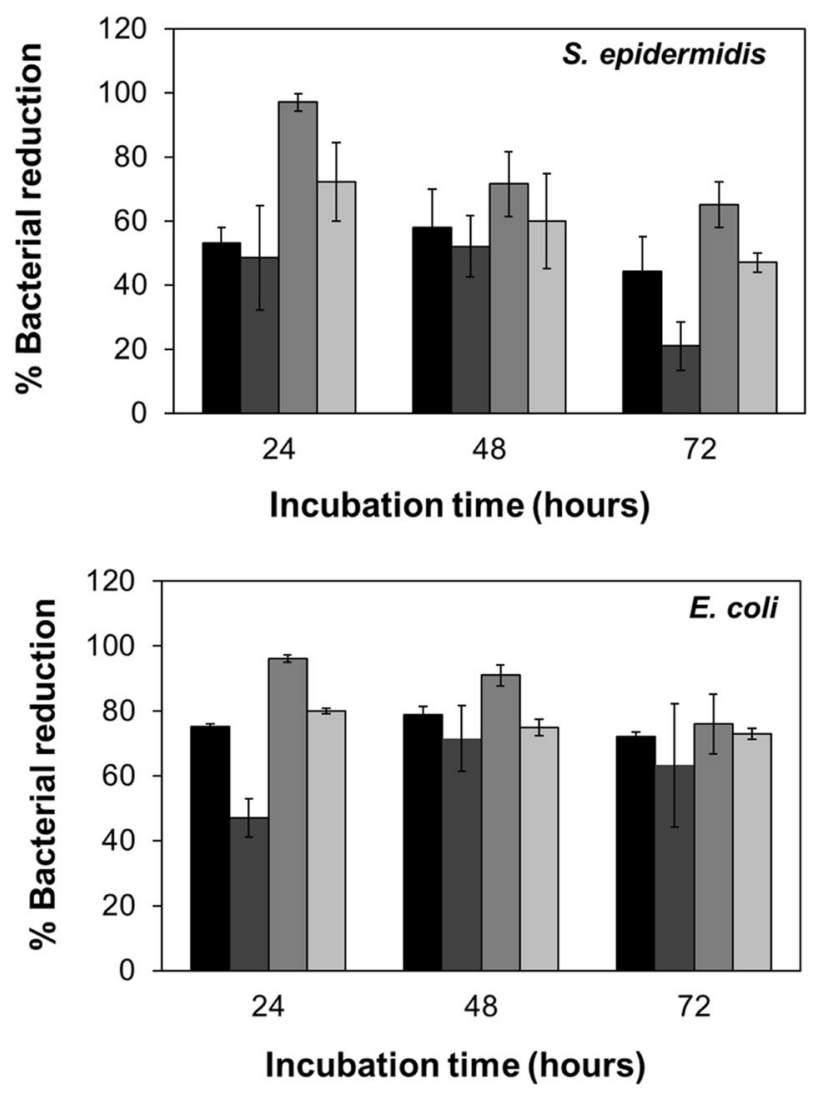

- Lubricant with poly(hydroxybenzene)

- Lubricant with poly(catechin)

口Lubricant wih poly(hydroxybenzene)-trimethoprim conjugate

口Lubricant with poly(catechin)-trimethoprim conjugate

Fig. 4 Bacterial reduction (\%) of S. epidermidis and E. coli when exposed to polyphenolic lubricant formulations. Four samples were tested: (1) lubricant-containing poly(hydroxybenzene) resulted from methylparaben oxidation by tyrosinase, (2) lubricant-containing poly(catechin) resulted from catechin oxidation by laccase, (3) lubricant-containing poly(hydroxybenzene)-trimethoprim conjugates obtained by in situ enzymatic oxidation of methylparaben and trimethoprim using tyrosinase, and (4) lubricant-containing poly(catechin)-trimethoprim conjugates produced in two operational steps as described by Gonçalves et al. (2015). Each assay was performed at $37^{\circ} \mathrm{C}$ for 24,48 , and $72 \mathrm{~h}$

by tyrosinase action. At that time, the formation of poly(hydroxybenzene) chains is expected since the tyrosinase-catalyzed oxidation of monohydric phenols involves the $o$-hydroxylation followed by the oxidation of so formed dihydric phenol units to the corresponding $o$-quinones which are highly reactive intermediates that can undergo nonenzymatic reactions as polymerization (Sousa et al. 2009). MALDI-TOF MS analysis allowed identifying $\mathrm{m} / \mathrm{z}$ peaks related with oligo- and poly(hydroxybenzene) compounds after tyrosinase-catalyzed methylparaben oxidation. The smallest oligomer was constituted at least by five repeating phenol units, while the highest molecular weight polymer was constituted by 37 repeating phenol units. Other by-products derived from this enzymatic reaction as benzoic acid and $p$-hydroxybenzoic acid were also detected on the mass spectrum (data not shown).

The enzymatic oxidation of methylparaben by tyrosinase was also tested in the presence of trimethoprim with the concomitant conjugation of the last at the reactive sites of oxidized methylparaben. The oxidation, biodegradation, and metabolism of trimethoprim are well described in literature (Michael et al. 2012). The major transformation steps include three pathways, namely cleavage, hydroxylation, and demethylation reactions. In general, the two-ring trimethoprim structure is maintained, and the major modifications are in the trimethoxybenzyl moiety. Demethylation/hydroxylation reactions can occur in parallel resulting in protonated molecules that can suffer hydroxyl radical attack forming different hydroxylated compounds. The trimethoprim cleavage on the left side of the central methylene group also yields low molecular weights products that can be further transformed by an oxidative opening of the aromatic ring. According to literature (Mallikarjunaswamy et al. 2013; Mohamed et al. 2010), the trimethoprim cleavage preserves the pyrimidine structure that is known to exhibit a range of pharmacological activity such as antibacterial and antifungal effects. As pyrimidine derivatives also possess a wide variety of potentially biological properties as antimicrobial activity, it is our assumption that the pyrimidine resulted from the trimethoprim cleavage conjugated with a polyphenolic chain allows producing a novel biologically active compound. At this point, it was our assumption that the poly(hydroxybenzene) units derived from the methylparaben oxidation by tyrosinase could be conjugated with trimethoprim or with trimethoprim oxidation by-products. Besides the presence of unchanged trimethoprim molecules, MALDI-TOF MS analysis allowed to confirm the in situ enzymatic poly(hydroxybenzene)-trimethoprim conjugation being identified trihydroxybenzene and hexahydroxybenzene chains coupled with trimethoprim molecule and its 2,4diaminopyrimidine-5-carboxylic acid oxidation by-product, respectively. Therefore, the in situ enzymatic conjugation of poly(hydroxybenzene) with trimethoprim was successfully achieved.

The rheological behavior of hydrophilic formulations, e.g., lubricants, used in pharmaceutical formulations is crucially important since it will directly affect the pathway of formulation development, the quality of raw and finished product, the components efficiency, the patient's compliance, and the overall healthcare costs (Mastropietro et al. 2013). In this work, the developed lubricant formulation was mainly composed by HEC and glycerol to confer the gelling/thickening and lubricating properties required. HEC is a nonionic hydrophilic, inexpensive, and nontoxic biodegradable natural biopolymer derived from cellulose. It is often used as a thickener in food, 
cosmetic, and pharmaceutical products (Beheshti et al. 2008; Velickova et al. 2009). When introduced into an aqueous medium, this compound increases the viscosity level. Glycerol belongs to the alcohol family of organic compounds and is a clear, colorless, viscous, and cheap raw material. It is highly recommended for the development of surfactants, lubricants, cosmetics, food additives, pharmaceuticals, personal care products, and industrial applications. An advantage of glycer$\mathrm{ol}$ is the ability to highly influence the solution viscosity. Depending on the concentration of glycerol present in the solution, it is possible to obtain a solution with a high or low viscosity. Besides, the percentage of water can influence the friction coefficient at the boundary and the elastohydrodynamic lubrication (Gholami et al. 2014; Shi et al. 2014). The developed lubricant formulations can be classified as pseudoplastic materials due to their shearthinning behavior, i.e., their viscosity decreases with the shear rate increase (Andrews et al. 2005; Rowe et al. 2006; Soares et al. 2011). This behavior can be attributed to the aggregation of individual particles that resulted from the break of intermolecular forces and further align in the direction of increasing shear (Cui et al. 1996; Rowe et al. 2006). The most concentrated formulation $(2.8: 11.1 \% \mathrm{HEC} /$ glycerol, $w / v)$ presented the higher viscosity level for all the shear rate values tested due to the intermolecular associations amongst the polymer chains leading to a much more compact network (Rowe et al. 2006; Soares et al. 2011). Moreover, this formulation was sensitive to temperature changes related with the HEC included in the lubricant. Indeed, as described in literature (Rowe et al. 2006; Savage 1957; Shin and Cho 1993), an increase of the temperature results in a reduction of the aqueous HEC solutions viscosity.

As previously mentioned, the two-step enzymatic synthesis of poly(catechin)-trimethoprim via laccase oxidation reported by Gonçalves et al. (2015) was also assessed in this work aiming to compare the antimicrobial efficiency of two polyphenol-antibiotic conjugates. Therefore, both enzymatically synthesized poly(hydroxybenzene)- and poly(catechin)-trimethoprim conjugates were introduced in the optimized lubricant formulation. Rheological tests showed that the lubricantcontaining poly(hydroxybenzene)- and poly(catechin)-trimethoprim conjugates maintained its non-Newtonian behavior; however, the initial viscosity values were slightly reduced. These results are a good indication that the developed formulations can be used for pharmacological proposes, as none of the compounds added to the formulation have promoted significant changes on its initial rheological properties (das Neves and Bahia 2006; Richardson et al. 2013).

When the lubricant formulations containing both produced polyphenolic compounds, poly(catechin) and poly(hydroxybenzene), were inoculated with $E$. coli and $S$. epidermidis, the bacterial reduction was higher in the first $24 \mathrm{~h}$ of incubation, due to a burst release of the antibacterial compounds from the lubricant matrix. After this period, it was expected the decrease of antibacterial compounds release and, therefore, the bacterial reduction also decreased. It is also noteworthy that a period for the lubricant dissolution is necessary to disrupt the barrier offered by the gel matrix and initiate the release of the compounds. Only after the lubricant dissolution, it was observed the maximum bacterial effect of each developed antimicrobial phenol. However, this behavior was not observed for the polyphenol-trimethoprim conjugates which can be attributed to the antibiotic action. The presence of free trimethoprim molecules in the polymeric mixture (Table 2) that easily diffuse through the lubricant matrix and subsequently promote bacterial damage masks the release of the polyphenol conjugates. After $72 \mathrm{~h}$ of incubation, all the developed compounds presented a decrease in the bacterial reduction which can be correlated with an increase in cell tolerance to the antimicrobial substances. As it is well described (Heipieper et al. 1994), one major adaptive mechanism of bacterial cells to the presence of phenols is to increase the degree of saturation of membrane lipids, therefore reducing the bacterial cell membrane damage. Comparing the antimicrobial efficiency of poly(hydroxybenzene), poly(catechin), poly (hydroxybenzene)-, and poly(catechin)trimethoprim conjugates incorporated into a lubricant matrix, it can be concluded that the tyrosinase methylparaben oxidation in the presence of trimethoprim enhanced the ability to reduce the bacterial growth. One of the reasons that can explain this result is the different structural conformation of each polyphenolic chain. Gonçalves et al. (2014) reported that the catechin oxidation yields a ramified polymer once on this work it was well expressed that the methylparaben oxidation leads to a more simple polymer constituted by repeating phenol units. This structure will have the phenol units more accessible to affect the structure of the bacterial cell wall. Another interesting phenomenon that can be responsible for the high antimicrobial activity of poly(hydroxybenzene)-trimethoprim conjugates synthesized by tyrosinase is the trimethoprim oxidation by-product, namely 2,4-diaminopyrimidine5 -carboxylic acid that is positively charged. As it is well described (Carmona-Ribeiro and de Melo Carrasco 2013), cationic compounds are emerging as promising candidates for the development of antimicrobial agents with reduced bacterial resistance since they are able to damage the bacterial cell through various action mechanisms such as adsorption and penetration into the cell wall, reaction with the cytoplasmic membrane (lipid or protein) followed by membrane disorganization, leakage of intracellular low-molecular-weight material, degradation of proteins and nucleic acids, and wall lysis caused by autolytic enzymes. Nevertheless, these polyphenol-trimethoprim conjugates offer the combination of two possible antimicrobial mechanisms such as the lipid bilayers perturbation and the intramembranous material leakage with consequent aggregation provided from the phenolic 
action and the dihydrofolate reductase inhibition caused by trimethoprim (Gonçalves et al. 2015) resulting in a higher inhibition of the bacterial growth. In addition, the polyphenols hydrophobicity considerable changes membrane functioning and influences protein-to-lipid ratios in the membrane, leading to the bacterial cells damage (Heipieper et al. 1994; Sikkema et al. 1995).

This work introduced a novel one-step enzymatic approach to produce antimicrobial poly(hydroxybenzene)-antibiotic conjugates. Methylparaben and trimethoprim were in situ oxidized through tyrosinase action leading to the formation of poly(hydroxybenzene)-trimethoprim conjugates. The incorporation of the poly(hydroxybenzene)-trimethoprim conjugates into a lubricant formulation did not compromised their rheological behavior and prompted antimicrobial activity against $E$. coli and $S$. epidermidis. Therefore, the in situ enzymatic conjugation of methylparaben and trimethoprim using tyrosinase as biocatalyst proved to be a great eco-friendly alternative approach to extend the methylparaben bacterial spectrum and to overcome the microbial resistance problems.

Acknowledgments The authors Idalina Gonçalves and Cláudia Botelho would like to acknowledge the NOVO project (FP7-HEALTH2011.2.3.1- 5) for funding. Loïc Hilliou acknowledges the financial support by FCT - Foundation for Science and Technology, Portugal (501100001871), through Grant PEst-C/CTM/LA0025/2013 - Strategic Project - LA 25 - 2013-2014, and by Programa Operacional Regional do Norte (ON.2) through the project "Matepro - Optimizing Materials and Processes", with reference NORTE-07-0124-FEDER-000037 FEDER COMPETE.

\section{References}

Alberto MR, Canavosio MAR, Nadra MCM (2011) Antimicrobial effect of polyphenols from apple skins on human bacterial pathogens. Electron J Biotechnol 9(3):205-209. doi:10.2225/vo19-issue3fulltext-1

Alkubati SA, Ahmed NT, Mohamed ONE, Fayed AM, Asfour HI (2015) Health care workers' knowledge and practices regarding the prevention of central venous catheter-related infection. Am J Infect Control 43(1):26-30. doi:10.1016/j.ajic.2014.09.021

Andrews GP, Gorman SP, Jones DS (2005) Rheological characterisation of primary and binary interactive bioadhesive gels composed of cellulose derivatives designed as ophthalmic viscosurgical devices. Biomaterials 26(5):571-580. doi:10.1016/j.biomaterials.2004.02. 062

Ba S, Haroune L, Cruz-Morató C, Jacquet C, Touahar IE, Bellenger J-P, Legault CY, Jones JP, Cabana H (2014) Synthesis and characterization of combined cross-linked laccase and tyrosinase aggregates transforming acetaminophen as a model phenolic compound in wastewaters. Sci Total Environ 487:748-755. doi:10.1016/j. scitotenv.2013.10.004

Beheshti N, Zhu K, Kjøniksen A-L, Nyström B (2008) Interaction behaviors in aqueous solutions of negatively and positively charged hydrophobically modified hydroxyethylcellulose in the presence of an anionic surfactant. Colloids Surf A Physicochem Eng Asp 328(1-3):79-89. doi:10.1016/j.colsurfa.2008.06.030
Błędzka D, Gromadzińska J, Wąsowicz W (2014) Parabens. From environmental studies to human health. Environ Int 67:27-42. doi:10. 1016/j.envint.2014.02.007

Capdevila JA (1998) Catheter-related infection: an update on diagnosis, treatment, and prevention. Int J Infect Dis 2(4):230-236

Carmona-Ribeiro A, de Melo Carrasco L (2013) Cationic Antimicrobial Polymers and Their Assemblies. Int J Mol Sci 14(5):9906-9946

Charnock C, Finsrud T (2007) Combining esters of para-hydroxy benzoic acid (parabens) to achieve increased antimicrobial activity. J Clin Pharm Ther 32(6):567-572. doi:10.1111/j.1365-2710.2007.00854.x

Cui ST, Gupta SA, Cummings PT, Cochran HD (1996) Molecular dynamics simulations of the rheology of normal decane, hexadecane, and tetracosane. J Chem Phys 105(3):1214-1220. doi:10.1063/1. 471971

das Neves J, Bahia MF (2006) Gels as vaginal drug delivery systems. Int J Pharm 318(1-2):1-14. doi:10.1016/j.ijpharm.2006.03.012

Decker H, Tuczek F (2000) Tyrosinase/catecholoxidase activity of hemocyanins: structural basis and molecular mechanism. Trends Biochem Sci 25(8):392-397. doi:10.1016/S0968-0004(00)01602-9

Demarche P, Junghanns C, Nair RR, Agathos SN (2012) Harnessing the power of enzymes for environmental stewardship. Biotechnol Adv 30(5):933-953. doi:10.1016/j.biotechadv.2011.05.013

Egbaria K, Friedman M (1990) Sustained in vitro activity of human albumin microspheres containing chlorhexidine dihydrochloride against bacteria from cultures of organisms that cause urinary tract infections. Antimicrob Agents Chemother 34(11):2118-2121

Faccio G, Kruus K, Saloheimo M, Thöny-Meyer L (2012) Bacterial tyrosinases and their applications. Process Biochem 47(12):1749 1760. doi:10.1016/j.procbio.2012.08.018

Frasca D, Dahyot-Fizelier C, Mimoz O (2010) Prevention of central venous catheter-related infection in the intensive care unit. Crit Care 14(2):212

Garg S, Tambwekar KR, Vermani K, Garg A, Kaul CL, Zaneveld LJ (2001) Compendium of pharmaceutical excipients for vaginal formulations. Pharm Technol 25(9; SUPP):14-25

Gholami Z, Abdullah AZ, Lee K-T (2014) Dealing with the surplus of glycerol production from biodiesel industry through catalytic upgrading to polyglycerols and other value-added products. Renew Sustain Energy Rev 39:327-341. doi:10.1016/j.rser.2014. 07.092

Gonçalves I, Matamá T, Cavaco-Paulo A, Silva C (2014) Laccase coating of catheters with poly(catechin) for biofilm reduction. Biocatalysis Biotransformation 32(1):2-12. doi:10.3109/10242422.2013. 828711

Gonçalves I, Abreu A, Matamá T, Ribeiro A, Gomes A, Silva C, CavacoPaulo A (2015) Enzymatic synthesis of poly(catechin)-antibiotic conjugates: an antimicrobial approach for indwelling catheters. Appl Microbiol Biotechnol 99(2):637-651. doi:10.1007/s00253014-6128-2

Heipieper HJ, Weber FJ, Sikkema J, Keweloh H, de Bont JAM (1994) Mechanisms of resistance of whole cells to toxic organic solvents. Trends Biotechnol 12(10):409-415

Kumon H, Hashimoto H, Nishimura M, Monden K, Ono N (2001) Catheter-associated urinary tract infections: impact of catheter materials on their management. Int J Antimicrob Agents 17(4):311-316

Mallikarjunaswamy C, Bhadregowda D, Mallesha L (2013) Synthesis and antimicrobial activity of pyrimidine salts with chloranilic and picric acids. J Chem. doi:10.1155/2013/727182

Mastropietro DJ, Nimroozi R, Omidian H (2013) Rheology in pharmaceutical formulations-A perspective. J Dev Drugs

Michael I, Hapeshi E, Osorio V, Perez S, Petrovic M, Zapata A, Malato S, Barceló D, Fatta-Kassinos D (2012) Solar photocatalytic treatment of trimethoprim in four environmental matrices at a pilot scale: transformation products and ecotoxicity evaluation. Sci Total Environ 430:167-173. doi:10.1016/j.scitotenv.2012.05.003 
Mielech-Łukasiewicz K, Puzanowska-Tarasiewicz H, Niedzielko A (2011) Electrooxidation of Some Antifungal Agents and Their Square-Wave Voltammetric Determination in Cosmetics and Pharmaceutics. Anal Lett 44(6):955-967. doi:10.1080/00032719. 2010.506934

Mohamed MS, Awad SM, Sayed AI (2010) Synthesis of Certain Pyrimidine Derivatives as Antimicrobial Agents and AntiInflammatory Agents. Molecules 15(3):1882-1890

Naik KM, Nandibewoor ST (2013) Spectral characterization of the binding and conformational changes of bovine serum albumin upon interaction with an anti-fungal drug, methylparaben. Spectrochim Acta A Mol Biomol Spectrosc 105:418-423. doi:10.1016/j.saa. 2012.12.055

Richardson BA, Kelly C, Ramjee G, Fleming T, Makanani B, Roberts S, Musara P, Mkandawire N, Moench T, Coletti A (2013) Appropriateness of hydroxyethylcellulose gel as a placebo control in vaginal microbicide trials: a comparison of the two control arms of HPTN 035. J Acquir Immune Defic Syndr (1999) 63(1):120

Rowe RC, Sheskey PJ, Owen SC, Association AP (2006) Handbook of pharmaceutical excipients, vol 6. Pharmaceutical press, London

Savage A (1957) Temperature-viscosity relationships for water-soluble cellulose ethers. Ind Eng Chem 49(1):99-103

Shi Y, Minami I, Grahn M, Björling M, Larsson R (2014) Boundary and elastohydrodynamic lubrication studies of glycerol aqueous solutions as green lubricants. Tribol Int 69:39-45. doi:10.1016/j. triboint.2013.08.013

Shin S, Cho YI (1993) Temperature effect on the non-Newtonian viscosity of an aqueous polyacrylamide solution. Int Commun Heat Mass Transf 20(6):831-844

Sikkema J, de Bont JA, Poolman B (1995) Mechanisms of membrane toxicity of hydrocarbons. Microbiol Rev 59(2):201-222

Snaterse M, Rüger W, Scholte op Reimer WJM, Lucas C (2010) Antibiotic-based catheter lock solutions for prevention of catheterrelated bloodstream infection: a systematic review of randomised controlled trials. J Hosp Infect 75(1):1-11. doi:10.1016/j.jhin. 2009.12.017
Soares LFJ, do Carmo F, de Almeida BV, Monteiro L, Rodrigues C, Cabral L, de Sousa V (2011) Preparation and evaluation of lidocaine hydrochloride in cyclodextrin inclusion complexes for development of stable gel in association with chlorhexidine gluconate for urogenital use. Int J Nanomedicine 6:1143-1154

Soni MG, Taylor SL, Greenberg NA, Burdock GA (2002) Evaluation of the health aspects of methyl paraben: a review of the published literature. Food Chem Toxicol 40(10):1335-1373. doi:10.1016/ S0278-6915(02)00107-2

Soni MG, Carabin IG, Burdock GA (2005) Safety assessment of esters of p-hydroxybenzoic acid (parabens). Food Chem Toxicol 43(7):9851015. doi:10.1016/j.fct.2005.01.020

Sousa F, Guebitz GM, Kokol V (2009) Antimicrobial and antioxidant properties of chitosan enzymatically functionalized with flavonoids. Process Biochem 44(7):749-756. doi:10.1016/j.procbio.2009.03. 009

Steter JR, Rocha RS, Dionísio D, Lanza MRV, Motheo AJ (2014) Electrochemical oxidation route of methyl paraben on a borondoped diamond anode. Electrochim Acta 117:127-133. doi:10. 1016/j.electacta.2013.11.118

Tada M, Kohno M, Kasai S, Niwano Y (2010) Generation mechanism of radical species by tyrosine-tyrosinase reaction. J Clin Biochem Nutr 47(2): 162

Tada M, Kohno M, Niwano Y (2014) Alleviation effect of arbutin on oxidative stress generated through tyrosinase reaction with Ltyrosine and L-DOPA. BMC Biochem 15(1):23

Velickova E, Winkelhausen E, Kuzmanova S, Cvetkovska M, Tsvetanov C (2009) Hydroxyethylcellulose cryogels used for entrapment of Saccharomyces cerevisiae cells. React Funct Polym 69(9):688693. doi:10.1016/j.reactfunctpolym.2009.05.002

Wang S-P, Chang C-L (1998) Determination of parabens in cosmetic products by supercritical fluid extraction and capillary zone electrophoresis. Anal Chim Acta 377(1):85-93. doi:10.1016/S00032670(98)00563-7

Xu D-Y, Yang Z (2013) Cross-linked tyrosinase aggregates for elimination of phenolic compounds from wastewater. Chemosphere 92(4): 391-398. doi:10.1016/j.chemosphere.2012.12.076 